also by diurnal time course. The litho- night. genecity of the bile was high in the mid-

\title{
(6) Basic and Clinical Studies on Bile Acid Preparations fot the Treatment of Gallstones
}

\author{
Goro Kajiyama, M D and Akira Maruhashi, M D
}

The First Department of Internal Medicine, Hiroshima University School of Medicine

\begin{abstract}
Although in clinical studies chenodeoxycholic acid (GDGA) is known to dissolve cholesterol gallstones, some of the recent results with experimental animals can be interpreted to suggest that it might be hepatotoxic or that it might even deteriorate the gallstone dissolution in bile.

To clarify factors for the side-effects and the deterioration caused by GDGA, its chemical dynamics and effects on the liver morphology in hamsters were studied. In addition, an attempt was made to correrate the radiographical appearance of stones with the effect of bile acid preparations, CDCA and its isomer, ursodeoxycholic acid (UDCA), in human subjects.
\end{abstract}

\section{METHODS}

\section{Animal experiments}

Hamsters were fed either normal or gallstone-provoking diet (GPD) with or without Kanamycin and given orally 5 or $50 \mathrm{mg} / \mathrm{kg} /$ day CDCA. Three weeks later, the animals were sacrified, and bile and blood were collected for the determination of bile acids and lipids. At the same time, the number of gallstones formed in the gallbladder was counted and liver samples were taken for the histological and enzymological examination. Several animals taken randomly from each group were used for the determination of bile acid pool size according to the washout technique ${ }^{1)} \cdot{ }^{14} \mathrm{C}$ CDCA were infused orally and radioactivity recovered in bile and blood was counted by liquid scintillation spectrophotometer. Liver function tests were performed by standard laboratory methods.

\section{Human trials}

Patients with cholelithiasis were given CDCA in doses of 100,300 and $450 \mathrm{mg}$ a day. Bile was collected before and three months after administration of the drug and analyzed to determine the lithogenic index. Sixty-six patients were classified according to the radiographical types of gallstone and given $300 \mathrm{mg}$ CDCA or UDCA per day for 3 to 24 months (average 14.3 months) after the collection of bile for the determination of lithogenic index.

Gallstones obtained by surgical operation were compared with preoperative radiographical appearance. Liver tissues from patients given CDCA or UDGA for more than one year were electromicroscopically examined.

\section{RDSULTS AND DISCUSSION}

Daily ingestion of $50 \mathrm{mg}$ CDCA increased the stone formation to some extent among hamsters fed a GPD, while the stone was not seen in hamsters fed a normal diet regardless of the ingestion of CDCA. The total primary bile acid pool decreased in the group given the GPD. In addition, the daily ingestion of CDCA increased CDCA pool and decreased cholic acid (CA) pool. These changes in the pool sizes were more in the group given $50 \mathrm{mg}$ than $5 \mathrm{mg}$ 
Change in chemical dynamics of bile and liver after administration of CDCA in hamstar.

Bile composition (Admirand \& Small)

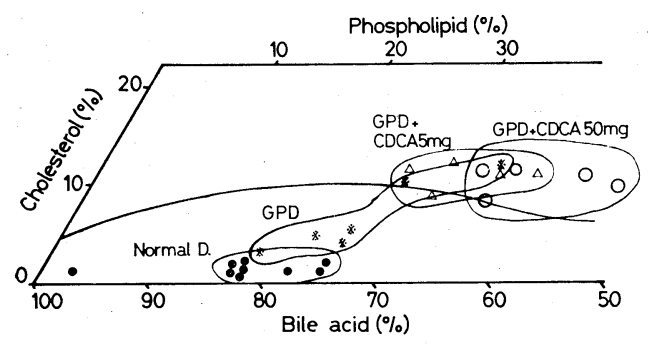

Lithogenic index, Serum bile acid, Bile acid pool and HMG-COA reductase activity in the liver

\begin{tabular}{|c|c|c|c|c|c|}
\hline & Normal Diet & GPD & $\begin{array}{l}\text { GPD, } \\
\text { CDCA5mg }\end{array}$ & $\begin{array}{c}\text { GPD. } \\
\text { CDCA50mg }\end{array}$ \\
\hline \multicolumn{2}{|c|}{ Lithogenic index } & $0.15 \div 0.07$ & $0.67 \pm 0.39$ & $1.21 \pm 0.17$ & $1.36 \pm 0.31$ \\
\hline \multirow{2}{*}{\multicolumn{2}{|c|}{ 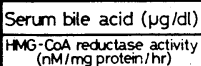 }} & $269 \pm 43$ & $503 \div 104$ & $359 \pm 20$ & $2700 \pm 1305$ \\
\hline & & $7.56 \pm 2.22$ & $27.12 \pm 10.02$ & & $14.34 \div 5.34$ \\
\hline \multirow{3}{*}{$\begin{array}{c}\text { Bile acid pool } \\
(\mu g)\end{array}$} & $\mathrm{CA}$ & $1902 \pm 1260$ & $878 \pm 677$ & $440 \pm 104$ & $314 \pm 77$ \\
\hline & CDCA & $558 \pm 325$ & $431 \pm 164$ & $941 \pm 128$ & $1191 \div 449$ \\
\hline & Total & $2435 \pm 1518$ & $1308 \pm 823$ & $1379 \pm 224$ & $1501 \pm 510$ \\
\hline
\end{tabular}

Monophological changes of the liver.

\begin{tabular}{|c|c|c|c|c|c|c|}
\hline & \multicolumn{2}{|c|}{ Normal Diet } & \multicolumn{3}{|c|}{ Gallstone Provoking Diet } \\
\hline & & Control & CDCA50mg & Control & COCA5OMg & $\begin{array}{l}\text { CDCA50my } \\
\text { Kananycin }\end{array}$ \\
\hline \multirow{2}{*}{ Liver cell } & Acidonhilic degeneration & - & \# & - & H & \# \\
\hline & Vacuole & - & + & - & \# & - \\
\hline \multicolumn{2}{|c|}{ Kupffer cell mobilzation } & - & + & - & + & + \\
\hline \multicolumn{2}{|c|}{ Destruction of limiting plate } & - & - & - & \# & + \\
\hline \multirow{4}{*}{$\begin{array}{l}\text { Glisson's } \\
\text { sheath }\end{array}$} & Ductular proliteration & - & H & - & H+ & + \\
\hline & Inflammatory cell & - & + & + & \# & H \\
\hline & Edema & - & - & - & + & + \\
\hline & \begin{tabular}{|l} 
Fibrosis \\
\end{tabular} & - & + & - & + & - \\
\hline
\end{tabular}

-: None +:Occasional H: Moderate HIt:Marked

CDCA. Total primary bile acid pool increased in both groups, given 5 and $50 \mathrm{mg}$ CDCA, in comparison to the control group, fed the GPD alone.

However, the increase of cholesterol in bile did overcome the increase of bile acid pool in hamsters given $50 \mathrm{mg}$ CDCA with the GDP, and resulted in an expansion of lithogenesity.

High HMG Co-A reductase and 7 alphahydroxylase activity caused by GPD was inhibited by daily ingestion of $50 \mathrm{mg}$ GDCA. The fact that the inhibition of the latter enzyme exceeded that of the former after ingestion of $50 \mathrm{mg}$ GDCA may explain the high production of cholesterol in the liver of this group.
Effect of CDCA and UDCA on gallstone dissolution.

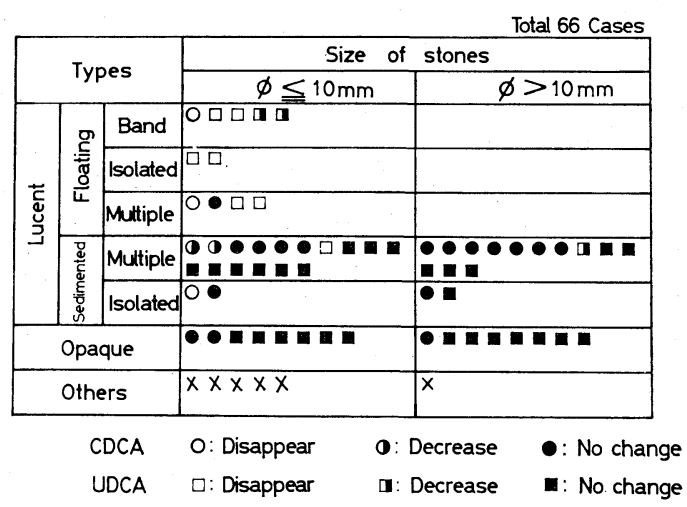

The pathological changes of the liver, including acidophilic degeneration and vacuole formation of liver cells, infiltration of inflammatory cells, edema and fibrosis in portal triads and destruction of limiting plate, were seen in the group given GDCA regardless of the diet. However, the more lesions were seen in the group given the GPD with the high dose of CDCA in comparison to the group given the normal diet with CDCA or the group given the GPD with the low dose of CDCA. These morphological changes of the liver were inhibited by the administration of Kanamycin together with the food and drinking water. This suggests that the combination of Kanamycin blocks the development of the hepatic toxicity by inhibiting the bacterial dehydroxylation of GDCA in the intestine to the form lithocholic acid ( $\mathrm{LAC}$ ) and that direct action of CDCA itself does not make a large contribution to the morphological changes of the liver. In conclusion with the above finding, the fact that LCA in bile was increased by ingestion of GDCA may indicate that LCA derived from CDCA causes the liver injury.

Together with the changes in the liver morphology, a drastic rise in fasting serum bile acid concentration ( $\mathrm{CA}$ as well as CDCA) was observed. The increased serum CA level, less radioactivity of ${ }^{14} \mathrm{C}$-GDGA in bile and high accumulation of radioactivity in the serum seems to indicate that the high accumulation of serum bile acid 
is due to less incorporation of bile acids into the injured liver than the intract liver, rather than an excess supply and absorption of GDCA through the intestine.

According to the recent reports, only $17 \%$ of LGA in the enterohepatic circulation is subjected to sulfation in the liver of rhesus mondey ${ }^{2}$ while in the human liver $60 \%$ of LGA can be sulfated ${ }^{32}$. These facts suggest that conflicating results in the effects of CDGA between animals and human came from the difference in the magnitude, such as of the inhibition of enzyme activities or of the sulfation of LCA, and not from the difference in direction of GDGA metabolism between animals and human. Therefore, in conclusion, when the less dose is employed for the treatment of cholelithiasis, the less often side-effects or unexpected affairs are encountered in the daily medication.

In human trials, $300 \mathrm{mg}$ or more GDCA improved the lithogenesity of bile in patients with cholelithiasis. Therefore, mainly $300 \mathrm{mg}$ dose of CDCA and UDCA was adopted in the treatment of cholelithiasis.

Stone dissolution was seen both floating and sedimented stones radiographically classified. In radiolucent stones, 90\% of floating stones and $22 \%$ of sedimented stones
( $\leqq 10 \mathrm{~mm}$ diameter) became smaller or disappeared by treatment with GDCA or UDCA. None of the radiopaque stones revealed any change indicating the decrease in size or number of stones.

The extent of the effect of CDCA and UDGA may depends on the quality and the size of the stones. Gallstones obtained by surgical operation showed that the majority of the radiolucent floating stones were pure cholesterol or cholesterol mixed stones while a half of the lucent sedimented stones was mixed stones and remainders were pigment or combination stones covered by Ca-bilirubinate. Almost all opaque stones were bilirubin-pigment stone. An increase in the dose of bile acid preparations may be effective to the lucent sedimented stones, but we should keep in mind that a half of them in pigment stones or stones covered by Ca-bilirubinate as mentioned above.

Electromicroscopic examination of the liver from patients given $300 \mathrm{mg}$ of CDGA or UDCA for more than one year showed no remarkable change.

\section{REFERENCES}

1) Mok HYI, et al: Gut 15:247, 1974.

2) Gadacz TR, et al: Gastroent $70: 1125,1976$.

3) Abstair E, et al : Gastroent 69:59, 1975.

\title{
Supplementary (1) Changes in Bile Acid Metabolism by Administration of Chenodeoxycholic Acid and Ursodeoxycholic Acid
}

\author{
Shoichi Nakagawa, M D and Isao Makino, M D \\ The Second Department of Medicine, Hakkaido University School of Medicine, Sapporo
}

In 1972, Danzinger et al reported that chenodeoxycholic acid (CDCA) induced cholesterol gallstone dissolution in man, and this effect has subsequently confirmed by a number of investigators. Ursodeoxycholic acid (UDGA) is the 7-beta epimer of GDGA, and this bile acid has been commonly used as cholagogue in Japan.

This report describes about changes of bile acid metabolism in bile, serum and feces by administration of GDCA and UDCA. 